\title{
Nonlinear Mechanical Properties of Graphene Nanoribbons
}

Qiang Lu and Rui Huang

Department of Aerospace Engineering and Engineering Mechanics, University of Texas, Austin, Texas 78712, USA

\begin{abstract}
Based on atomistic simulations, the nonlinear elastic properties of monolayer graphene nanoribbons under quasistatic uniaxial tension are predicted, emphasizing the effect of edge structures (armchair and zigzag, without and with hydrogen passivation). The results of atomistic simulations are interpreted using a theoretical model of thermodynamics, which enables determination of the nonlinear functions for the strain-dependent edge energy and the hydrogen adsorption energy, for both zigzag and armchair edges. Due to the edge effects, the initial Young's modulus of graphene nanoribbons under infinitesimal strain varies with the edge chirality and the ribbon width. Furthermore, it is found that the nominal strain to fracture is considerably lower for armchair graphene nanoribbons than for zigzag ribbons. Two distinct fracture mechanisms are identified, with homogeneous nucleation for zigzag ribbons and edgecontrolled heterogeneous nucleation for armchair ribbons.
\end{abstract}

\section{INTRODUCTION}

Graphene ribbons with nanoscale widths $(W<20 \mathrm{~nm})$ have been produced recently, either by lithographic patterning [1-3] or by chemically derived self assembly processes [4], with potential applications in nanoelectronics and electromechanical systems. The edges of the graphene nanoribbons (GNRs) could be zigzag, armchair, or a mixture of both [5]. It has been theoretically predicted that the special characteristics of the edge states leads to a size effect in the electronic state of graphene and controls whether the GNR is metallic, insulating, or semiconducting [5-8]. The effects of the edge structures on deformation and mechanical properties of GNRs have also been studied to some extent [9-18]. On one hand, elastic deformation of GNRs has been suggested as a viable method to tune the electronic structure and transport characteristics in graphene-based devices $[15,16]$. On the other hand, plastic deformation and fracture of graphene may pose a fundamental limit for reliability of integrated graphene structures.

The mechanical properties of bulk graphene (i.e., infinite lattice without edges) have been studied both theoretically [19-21] and experimentally [22]. For GNRs, however, various edge structures are possible $[23,24]$, with intricate effects on the mechanical properties. Ideally, the mechanical properties of GNRs may be characterized experimentally by uniaxial tension tests. To date however no such experiment has been reported, although similar tests were performed for carbon nanotubes (CNTs) [25]. Theoretically, previous studies on the mechanical properties of GNRs have largely focused on the linear elastic properties (e.g., Young's modulus and Poisson's ratio) [11-15]. While a few studies have touched upon the nonlinear mechanical behavior including fracture of GNRs $[12,13,16]$, the effect of the edge structures in the nonlinear regime has not been well understood. In the present study, based on atomistic simulations and a thermodynamics model, the nonlinear elastic deformation of graphene 
nanoribbons under quasistatic uniaxial tension are analyzed, emphasizing the effects of edge structures on elastic modulus and fracture strength.

\section{ATOMISTIC SIMULATIONS}

Atomistic simulations of GNRs under uniaxial tension are performed using the secondgeneration reactive empirical bond order (REBO) potential [26]. In each simulation, the tensile strain is applied incrementally in the longitudinal direction of the GNR, until fracture occurs. At each strain level, the statically equilibrium lattice structure of the GNR is calculated to minimize the total potential energy by a quasi-Newton algorithm. Periodic boundary conditions are applied at both ends of the GNR, whereas the two parallel edges (zigzag or armchair) of the GNR are free of external constraint. To study the effect of hydrogen passivation along the free edges, the results for GNRs with bare and passivated edges are compared. The mechanical behavior of infinite graphene lattice is also simulated by applying the periodic boundary conditions at all four edges, for which the uniaxial stress state is achieved by lateral relaxation perpendicular to the loading direction.
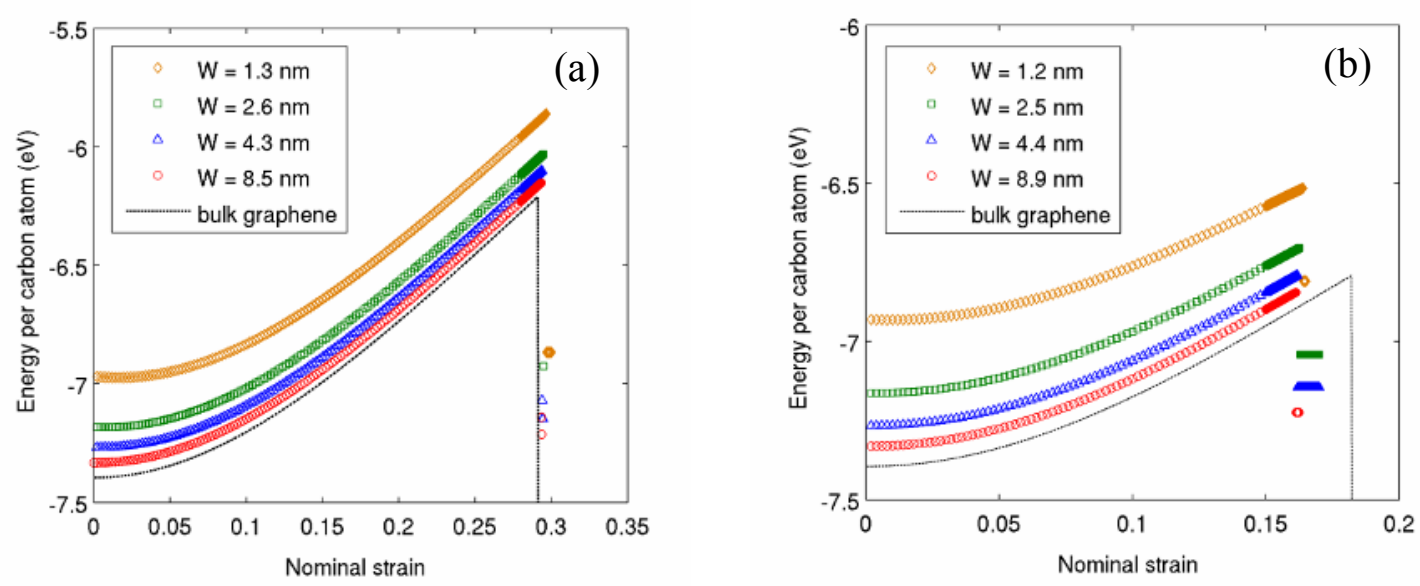

Figure 1. Potential energy per carbon atom as a function of the nominal strain for graphene nanoribbons under uniaxial tension, with (a) zigzag and (b) armchair edges, both unpassivated.

Figure 1 shows the results from atomistic simulations for GNRs with unpassivated edges, where the ribbon width $(W)$ is varied between 1 and $10 \mathrm{~nm}$. For each GNR, the average potential energy per carbon atom increases as the nominal strain increases until it fractures at a critical strain. To understand the numerical results, we adopt a simple thermodynamics model for the uniaxially stressed GNRs. For a GNR of width $W$ and length $L$, the total potential energy as a function of the nominal strain consists of contributions from deformation of the interior lattice (bulk strain energy) and from the edges (edge energy), namely

$$
\phi(\varepsilon)=\phi_{0} W L+U(\varepsilon) W L+2 \gamma(\varepsilon) L,
$$

where $\varepsilon$ is the nominal strain (relative to the bulk graphene lattice at the ground state), $\phi_{0}$ is the potential energy density (per unit area) of graphene at the ground state, $U(\varepsilon)$ is the bulk strain energy density (per unit area), and $\gamma(\varepsilon)$ is the edge energy density (per unit length of the edges). 
While the bulk strain energy density as a function of the nominal strain can be obtained directly from the atomistic calculations for the infinite graphene lattice (dashed lines in Fig. 1), the edge energy density function is determined by subtracting the bulk energy from the total potential energy of the GNRs based on Eq. (1). Thus, both the energy functions are atomistically determined, which can then be fitted with nonlinear polynomial functions for theoretical purposes [20].

The GNR under uniaxial tension is subjected to a net force $(F)$ in the longitudinal direction. At each strain increment, the mechanical work done by the longitudinal force equals the increase of the total potential energy, which can be written in a variational form, i.e.,

$$
\delta \phi=F L \delta \varepsilon
$$

Consequently, the force $(F)$ can be obtained from the derivative of the potential energy function in Eq. (1). A two-dimensional (2-D) nominal stress can then be defined without ambiguity as the force per unit width of the GNR, namely

$$
\sigma(\varepsilon)=\frac{F}{W}=\frac{d U}{d \varepsilon}+\frac{2}{W} \frac{d \gamma}{d \varepsilon} .
$$
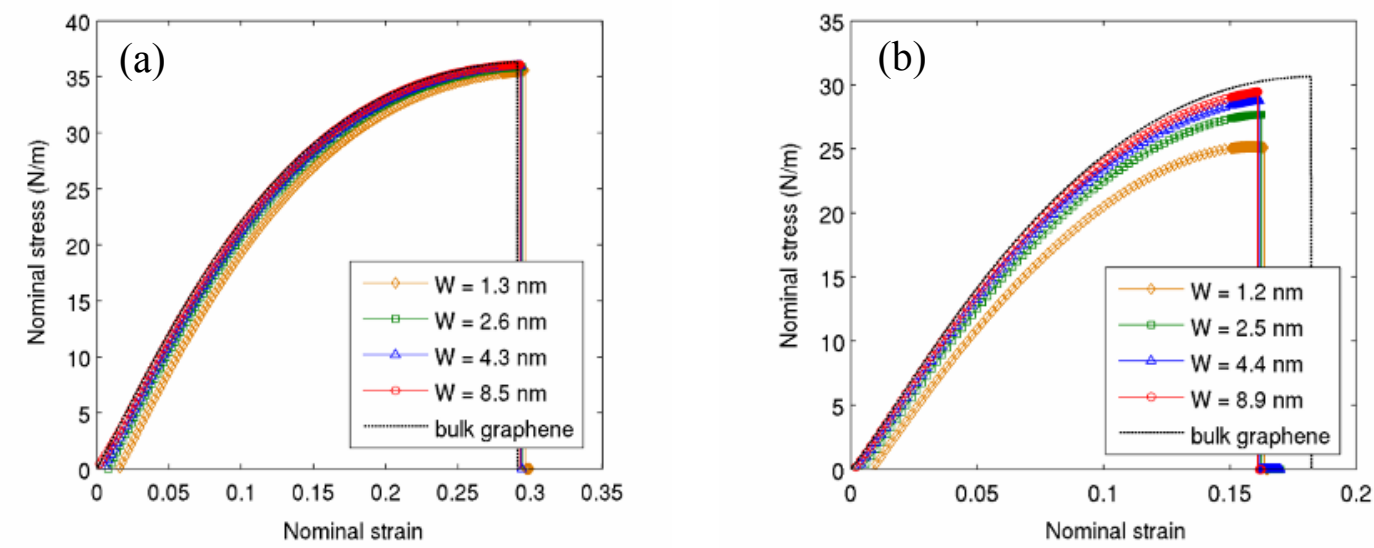

Figure 2. Nominal stress-strain curves for graphene nanoribbons under uniaxial tension, with (a) zigzag and (b) armchair edges, both unpassivated.

Figure 2 shows the 2-D nominal stress-strain curves of the GNRs obtained by taking the derivative of the potential energy curves in Fig. 1. Apparently, the stress-strain relation of a GNR is generally nonlinear, for which the tangent modulus as a function of the nominal strain is defined as

$$
E(\varepsilon)=\frac{d \sigma}{d \varepsilon}=\frac{d^{2} U}{d \varepsilon^{2}}+\frac{2}{W} \frac{d^{2} \gamma}{d \varepsilon^{2}}
$$

The first term on the right-hand side of Eq. (4) represents the tangent modulus of the bulk graphene lattice (under the condition of uniaxial stress), and the second term is the contribution from the edges (i.e., edge modulus). Therefore, the elastic modulus of the GNR in general depends on the ribbon width $(W)$ as well as the edge chirality. Similar stress-strain curves were 
obtained by molecular dynamics (MD) simulations [12], where the critical strain to fracture is typically lower than the static MM simulations due to the effects of temperature and loading rate.

\section{DISCUSSIONS}

The nominal stress-strain curves in Fig. 2 show approximately linear elastic behavior of all GNRs at relatively small strains (e.g., $\varepsilon<5 \%$ ). Following Eq. (4), the initial Young's modulus of the GNRs in the linear regime can be written as

$$
E_{0}=E_{0}^{b}+\frac{2}{W} E_{0}^{e}
$$

where $E_{0}^{b}$ is the initial Young's modulus of the bulk graphene and $E_{0}^{e}$ is the initial edge modulus. While the bulk graphene is isotropic in the regime of linear elasticity, the edge modulus depends on the edge chirality with different values for the zigzag and armchair edges. As a result, the initial Young's modulus of the GNR depends on the edge chirality and the ribbon width, as shown in Fig. 3. The REBO potential used in the present study predicts a bulk Young's modulus, $E_{0}^{b}=243 \mathrm{~N} / \mathrm{m}$, and the predicted edge modulus is $E_{0}^{e}=8.33 \mathrm{nN}(\sim 52 \mathrm{eV} / \mathrm{nm})$ for the unpassivated zigzag edge and $E_{0}^{e}=3.65 \mathrm{nN}(\sim 22.8 \mathrm{eV} / \mathrm{nm})$ for the unpassivated armchair edge. With positive moduli for both edges, the Young's modulus of unpassivated GNRs increases as the ribbon width decreases. We note that the predicted edge modulus is considerably lower than a previous calculation using a different potential [11], and the REBO potential is known to underestimate the bulk modulus $[27,28]$.

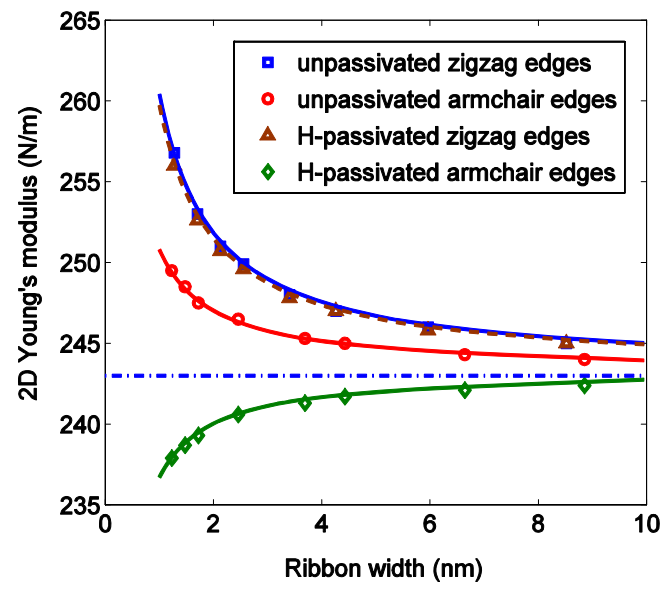

Figure 3. Initial Young's modulus versus ribbon width for GNRs with unpassivated and hydrogen-passivated edges.

For a GNR with hydrogen $(\mathrm{H})$ passivated edges, the potential energy in Eq. (1) is modified to account for the hydrogen adsorption, namely

$$
\phi(\varepsilon)=\phi_{0} W L+U(\varepsilon) W L+2 \gamma(\varepsilon) L-2 \gamma_{H}(\varepsilon) L
$$


where $\gamma_{H}(\varepsilon)$ is the adsorption energy of hydrogen per unit length along the edges of the GNR and the negative sign indicates typically reduced edge energy due to hydrogen passivation [17, 23]. By comparing the potential energies for the GNRs with and without H-passivation, the adsorption energy can be determined as a function of the nominal strain for both armchair and zigzag edges. At zero strain $(\varepsilon=0)$, our MM calculations predict the hydrogen adsorption energy to be 20.5 and $22.6 \mathrm{eV} / \mathrm{nm}$ for the zigzag and armchair edges, respectively, which compare closely with the first-principle calculations [23]. Under uniaxial tension, the adsorption energy varies with the nominal strain. Similar to Eq. (3), the nominal stress for the H-passivated GNR is obtained as

$$
\sigma(\varepsilon)=\frac{d U}{d \varepsilon}+\frac{2}{W}\left(\frac{d \gamma}{d \varepsilon}-\frac{d \gamma_{H}}{d \varepsilon}\right)
$$

The effect of hydrogen passivation on the initial Young's modulus of GNRs is shown in Fig. 3. Interestingly, while hydrogen passivation has negligible effect on the initial Young's modulus of GNRs with zigzag edges, the effect is dramatic for GNRs with armchair edges. In the latter case, a negative edge modulus ( $E_{0}^{e}=-20.5 \mathrm{eV} / \mathrm{nm}$ ) is obtained, and thus the initial Young's modulus decreases with decreasing ribbon width, opposite to the unpassivated GNRs.
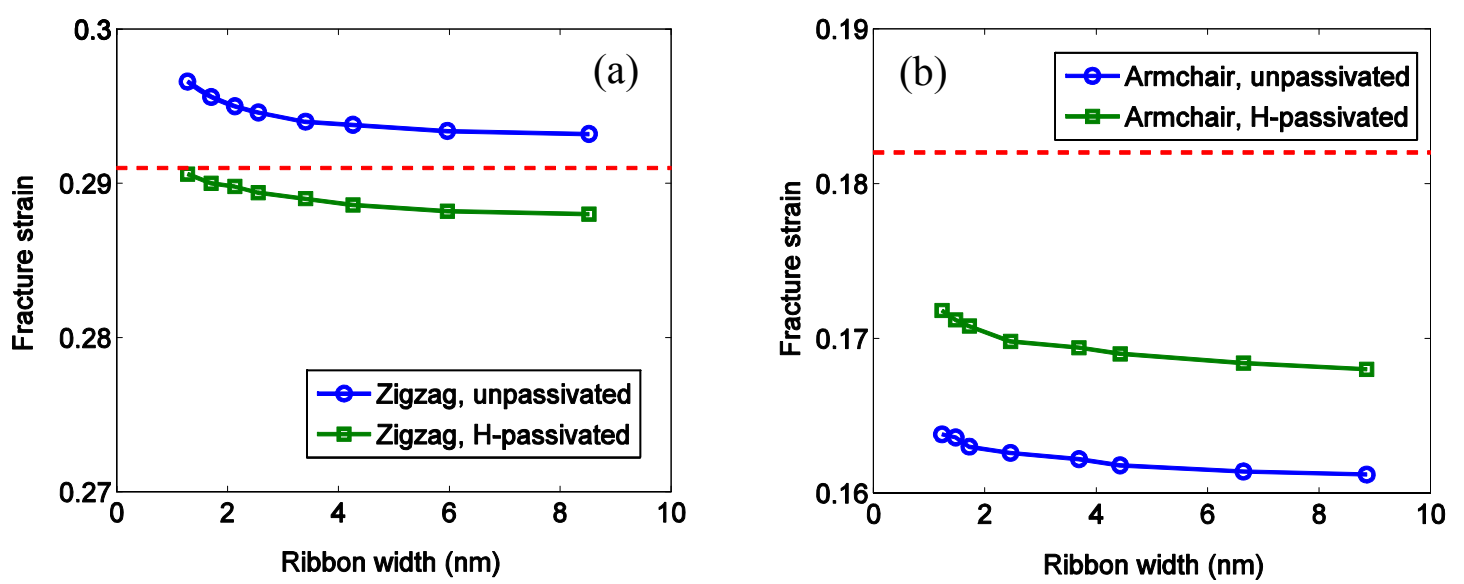

Figure 4. Fracture strain versus ribbon width for GNRs under uniaxial tension, with (a) zigzag and (b) armchair edges. The horizontal dashed line in each figure indicates the fracture strain of bulk graphene under uniaxial tension in the same direction.

Without any defect, the bulk graphene fractures when the tangent modulus becomes zero (i.e., $d^{2} U / d \varepsilon^{2}=0$ ), dictated by the intrinsic lattice instability under tension [19-21]. At a finite temperature, however, fracture may occur much earlier due to thermally activated processes [12]. As shown in a previous study [21], the critical strain to fracture for bulk graphene varies with the loading direction. Both first-principle calculations [19, 20] and empirical potential models [21] have predicted that the intrinsic critical strain is higher for graphene under uniaxial tension in the zigzag direction than in the armchair direction, suggesting that the hexagonal lattice of graphene preferably fractures along the zigzag directions by cleavage. As shown in Fig. 1a, the GNRs with zigzag edges fracture at a critical strain close to that of bulk graphene loaded in the same direction. In contrast, Fig. 1b shows that the GNRs with armchair edges fracture at a critical 
strain considerably lower than bulk graphene. In both cases, the fracture strain slightly depends on the ribbon width, as shown in Fig. 4. Hydrogen passivation of the edges leads to slightly lower fracture strains for zigzag GNRs, but slightly higher fracture strains for armchair GNRs.

The apparently different edge effects on the fracture strain imply different fracture mechanisms for the zigzag and armchair GNRs. The processes of fracture nucleation in GNRs are studied by molecular dynamics (MD) simulations at different temperatures. It is found that the edge effect leads to two distinct mechanisms for fracture nucleation in GNRs at relatively low temperatures $(T<300 \mathrm{~K})$. Figure 5 shows two fractured GNRs at $50 \mathrm{~K}$. For the GNR with zigzag edges (Fig. 5a), fracture nucleation occurs stochastically at the interior lattice of the zigzag GNRs. As a result, the fracture strain is very close to that of bulk graphene strained in the same direction, consistent with the MM calculations. However, for the GNR with armchair edges (Fig. 5b), fracture nucleation occurs exclusively near the edges. Thus, the armchair edge serves as the preferred location for fracture nucleation, leading to a lower fracture strain compared to bulk graphene, as seen also from the MM calculations. Therefore, two distinct fracture nucleation mechanisms are identified as interior homogeneous nucleation for the zigzag GNRs and edge-controlled heterogeneous nucleation for the armchair GNRs. The same mechanisms hold for GNRs with H-passivated edges. It is evident from Fig. 5 that cracks preferably grow along the zigzag directions of the graphene lattice in both cases.

(a)

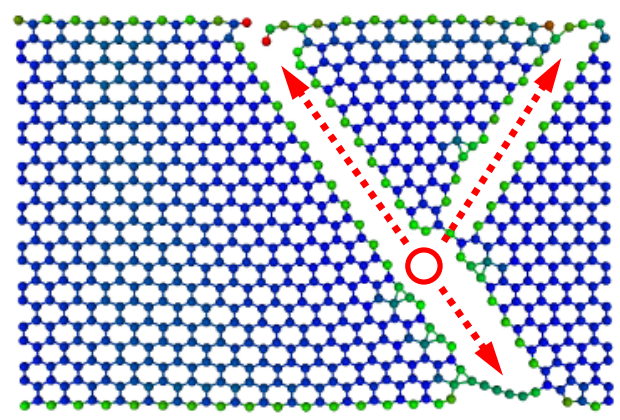

(b)

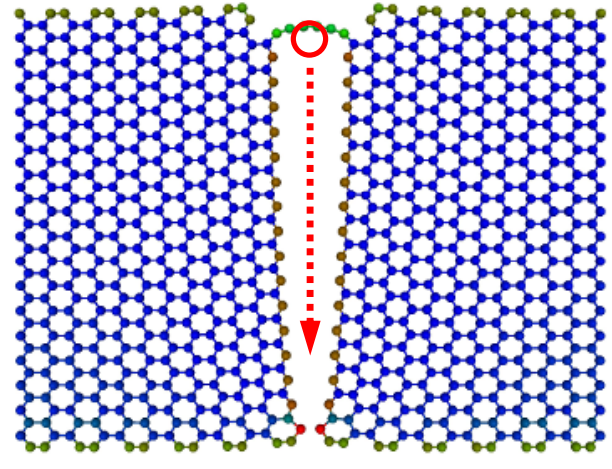

Figure 5. Fracture of graphene nanoribbons under uniaxial tension. (a) Homogeneous nucleation for a zigzag GNR; (b) edge-controlled heterogeneous nucleation for a armchair GNR. The circles indicate the nucleation sites, and the arrows indicate the directions of crack growth.

\section{CONCLUSIONS}

In summary, this paper presents a theoretical study on the effects of edge structures on the mechanical properties of graphene nanoribbons under uniaxial tension. Due to the edge effect, the initial Young's modulus of GNRs under infinitesimal strain depends on both the chirality and the ribbon width. Furthermore, it is found that the strain to fracture is considerably lower for armchair GNRs than that for zigzag GNRs. Two distinct fracture mechanisms are identified, with homogeneous nucleation for the zigzag GNRs and edge-controlled heterogeneous nucleation for the armchair GNRs. Hydrogen passivation is found to have relatively small effects on the mechanical behavior of zigzag graphene ribbons, but its effect is more significant for armchair ribbons. 


\section{ACKNOWLEDGMENTS}

The authors gratefully acknowledge funding of this work by National Science Foundation through Grant No. 0926851.

\section{REFERENCES}

1. C. Berger, et al., Science 312, 1191-1196 (2006).

2. B. Ozyilmaz, et al., Phys. Rev. Lett. 99, 166804 (2007).

3. Y. M. Lin, et al., Phys. Rev. B 78, 161409R (2008).

4. X. L. Li, et al., Science 319, 1229-1232 (2008).

5. K. Nakada, M. Fujita, G. Dresselhaus, M. S. Dresselhaus, Phys. Rev. B 54, 17954-17961 (1996).

6. Y.-W. Son, M. L. Cohen, S. G. Louie, Phys. Rev. Lett. 97, 216803 (2006).

7. V. Barone, O. Hod, G. E. Scuseria, Nano Lett. 6, 2748-2754 (2006).

8. S. Dutta, S. Lakshmi, S. K. Pati, Phys. Rev. B 77, 073412 (2008).

9. V. B. Shenoy, C. D. Reddy, A. Ramasubramaniam, Y. W. Zhang, Phys. Rev. Lett. 101, 245501 (2008).

10. K. V. Bets, B. I. Yakobson, Nano Research 2, 161-166 (2009).

11. C. D. Reddy, A. Ramasubramaniam, V. B. Shenoy, Y. W. Zhang, Appl. Phys. Lett. 94, 101904 (2009).

12. H. Zhao, K. Min, N. R. Aluru, Nano Lett. 9, 3012-3015 (2009).

13. H. Bu, et al., Phys. Lett. A 373, 3359-3362 (2009).

14. Z. P. Xu, J. Computational and Theoretical Nanoscience 6, 625-628 (2009).

15. R. Faccio, P. A. Denis, H. Pardo, C. Goyenola, A. W. Mombru, J. Phys.: Condens. Matter 21, 285304 (2009).

16. M. Topsakal, S. Ciraci, Phys. Rev. B 81, 024107 (2010).

17. C. K. Gan, D. J. Srolovitz, Phys. Rev. B 81, 125445 (2010).

18. Q. Lu, R. Huang, Phys. Rev. B 81, 155410 (2010).

19. F. Liu, P. M. Ming, J. Li, Phys. Rev. B 76, 064120 (2007).

20. X. Wei, B. Fragneaud, C. A. Marianetti, J. W. Kysar, Phys. Rev. B 80, 205407 (2009).

21. Q. Lu, R. Huang, Int. J. Appl. Mech. 1, 443-467 (2009).

22. C. Lee, X. Wei, J. W. Kysar, J. Hone, Science 321, 385-388 (2008).

23. P. Koskinen, S. Malola, H. Hakkinen, Phys. Rev. Lett. 101, 115502 (2008).

24. X. Jia, et al., Science 323, 1701-1705 (2009).

25. M. F. Yu, O. Lourie, M. J. Dyer, K. Moloni, T. F. Kelly, R. S. Ruoff, Science 287, 637-640 (2000).

26. D. W. Brenner, O. A. Shenderova, J. A. Harrison, S. J. Stuart, B. Ni, S. B. Sinnott, J. Phys. Condens. Mat. 14, 783-802 (2002).

27. M. Arroyo, T. Belytschko, Phys. Rev. B. 69, 115415 (2004).

28. J. Zhou, R. Huang, J. Mech. Phys. Solids 56, 1609-1623 (2008). 